\title{
Effect of Charge and Phosphine Ligands on the Electronic Structure of the Aus
}

\author{
Cluster Juan C. Burgos ${ }^{1,2^{*}}$, Sol M. Mejía ${ }^{2 *}$, and Gregory F. Metha ${ }^{3}$ \\ ${ }^{1}$ Programa de Ingeniería Química, Universidad de Cartagena, Cartagena, Colombia \\ ${ }^{2}$ Departamento de Química, Pontificia Universidad Javeriana, Bogotá, Colombia \\ ${ }^{3}$ Department of Chemistry, University of Adelaide, Adelaide SA 5005, Australia \\ Electronic_addresses: jburgosb@unicartagena.edu.co, sol.mejia@javeriana.edu.co \\ (corresponding author), and greg.metha@adelaide.edu.au.
}

Supporting Information 


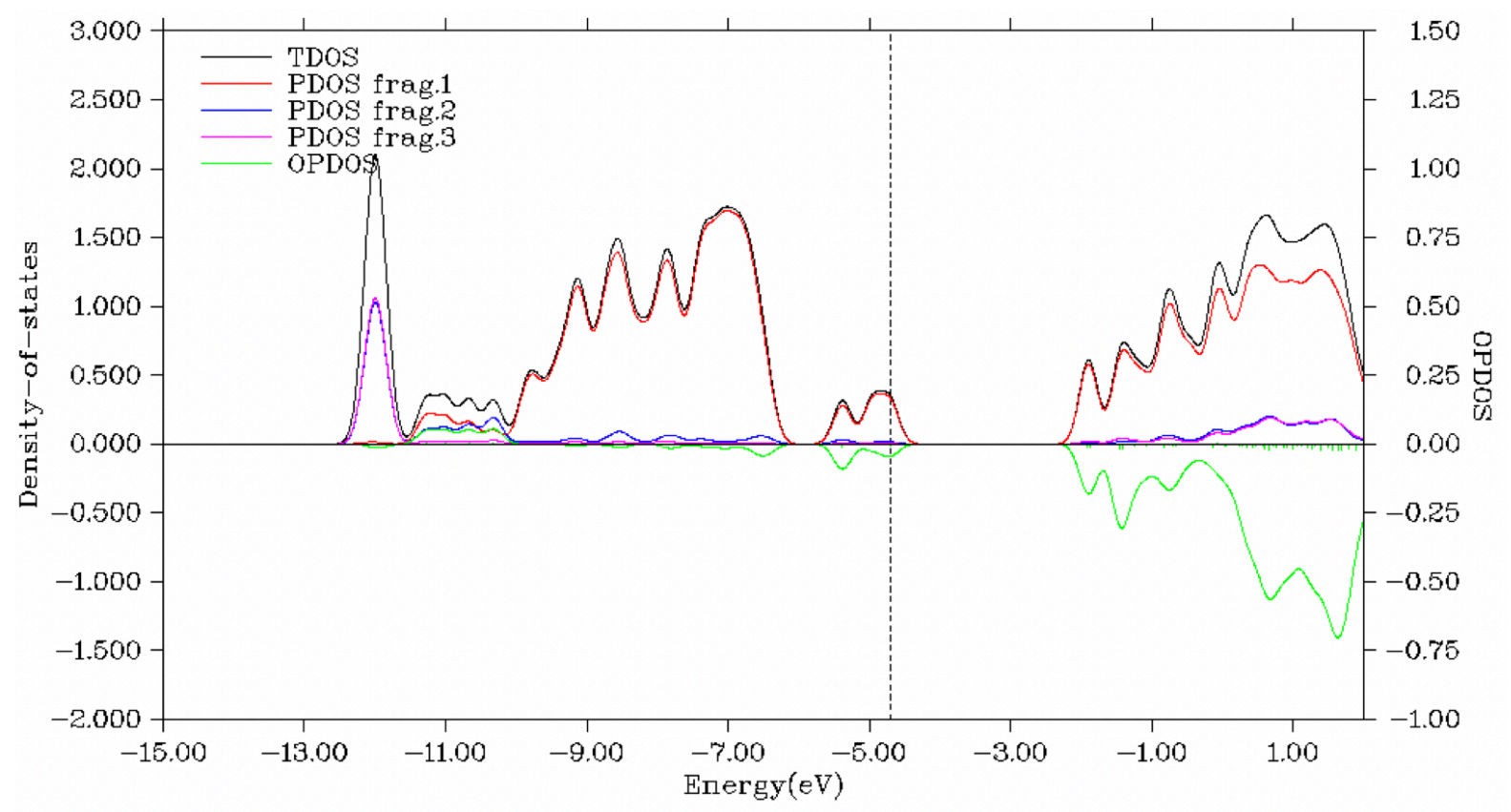

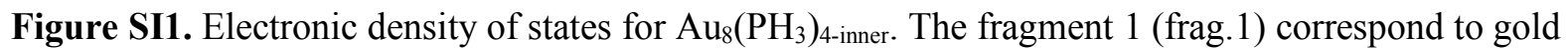
$(\mathrm{Au})$ atoms, frag.2 to phosphorous $(\mathrm{P})$, and frag.3 to hydrogens $(\mathrm{H})$. The OPDOS represents the overlap density of states between Au and $\mathrm{P}$ atoms.
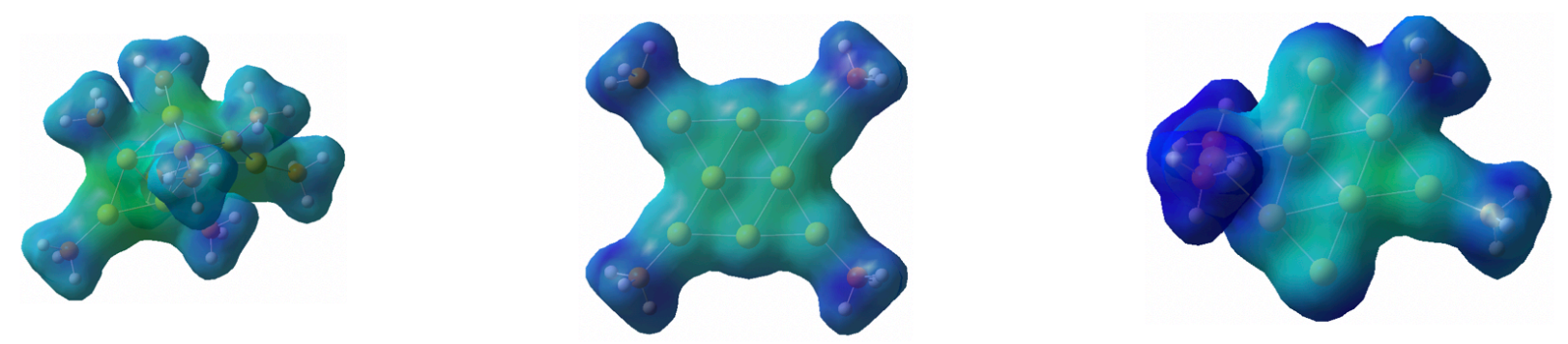

Figure SI2. Electrostatic potential mapped on the electron density surface of charged ligand protected clusters. 


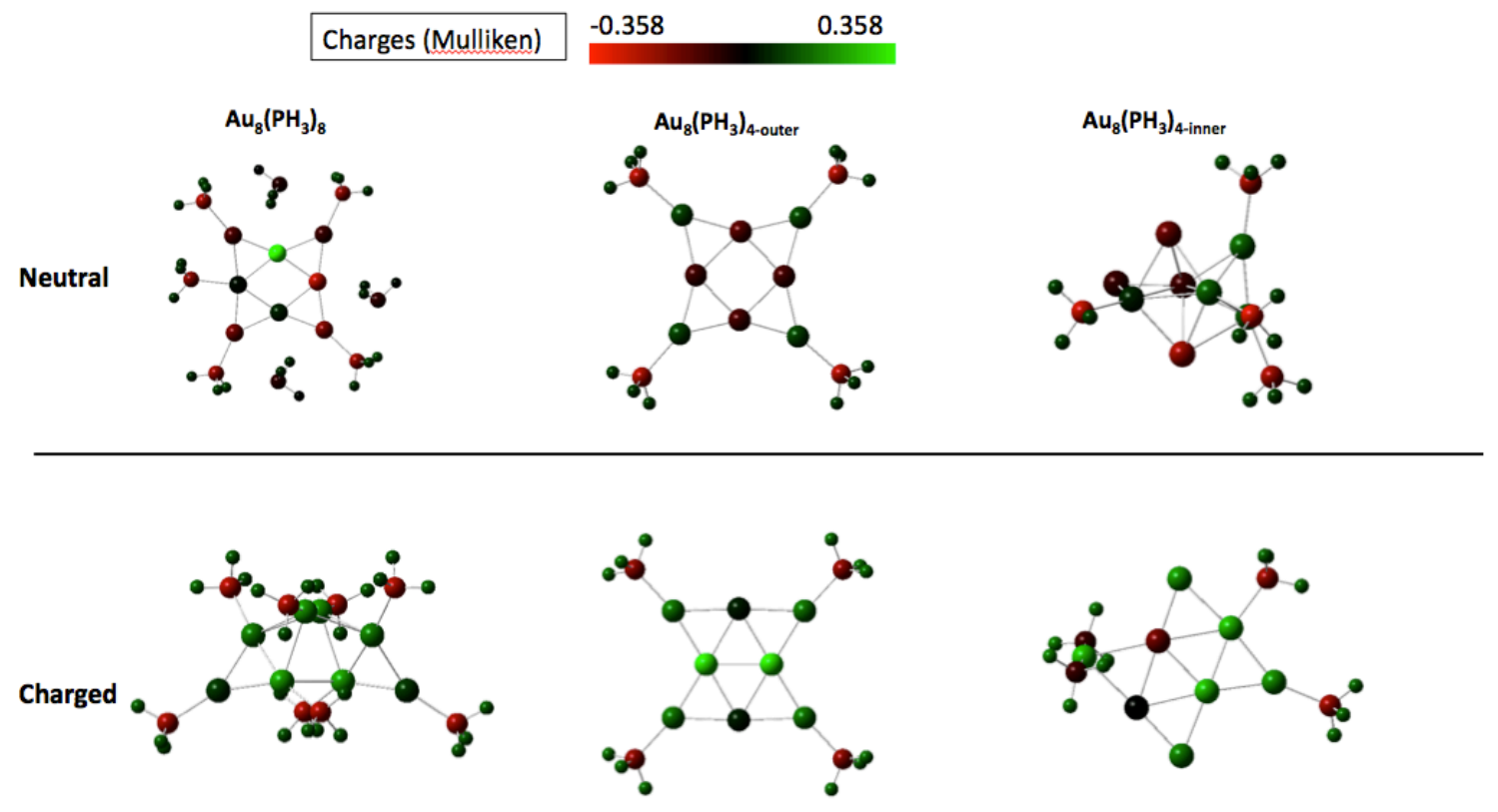

Figure SI3. Mulliken population analysis for neutral and charged ligand-protected clusters. 


\section{Kick code}

The version of the kick program employed in this study is the one published at the Journal of Computational Chemistry under the title: Kick: Constraining a stochastic search procedure with molecular fragments by Matthew A. Addicoat and Gregory F. Metha (REF: https://doi.org/10.1002/jcc.21026). This program is an extension of the Kick program developed by Bera et al. (J Phys Chem A 2006, 110, 4287). Nowadays, M. A. Addicoat has programmed a new version of this method called "CrazyLego" which also stochastically generates structures in combination with quantum mechanical methods but faster like Dispersion-corrected, third-order self-consistent-charge density-functional tight-binding (DFTB3). Its efficiency was demonstrated at the stochastic determination of the structures of an ionic liquid (10.1002/jcc.23420).

In the present case, for $\mathrm{Au}_{8}$ cluster, the eight gold atoms were specified and kicked into a box with a box length of $5 \AA$ (the dimensions of the box may be set independently for each problem). To solve this problem, in each job, each atom has its coordinates rotated by a random angle around each Cartesian axis and the new (rotated) coordinates are then translated (kicked) into the box. Batches of 70-100 jobs were run until no new isomers were identified. By default, no coordinates were frozen in the geometry optimization. This capability allows a stochastic search procedure. A separate parsing script determines all those jobs that have completed normally,

eliminates redundant jobs (energies within $1 \times 10^{-5}$ a.u.), orders the remaining energies and lists the Cartesian coordinates (also archive entries) of each unique structure. Finally, another auxiliary script is able to take all unique geometries and resubmit them either as frequency calculations or optimizations/single point energies at a higher level of theory calling the Gaussian program. In our case, we obtained the $\mathrm{Au}_{8}$ clusters with the multiplicities 1, 3 and 5.

As an example of an output of a batch of jobs with multiplicity $=1$ 


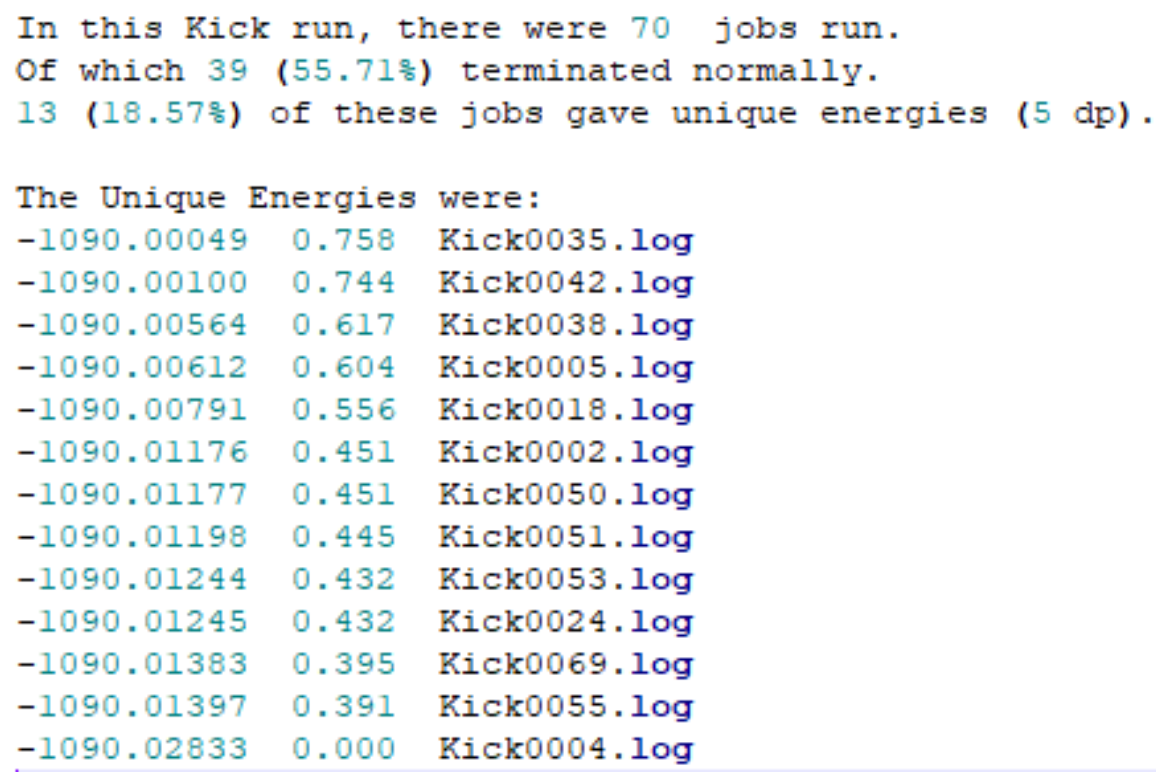

The three most stable structure with different multiplicities (Relative energy in $\mathrm{eV}$ )

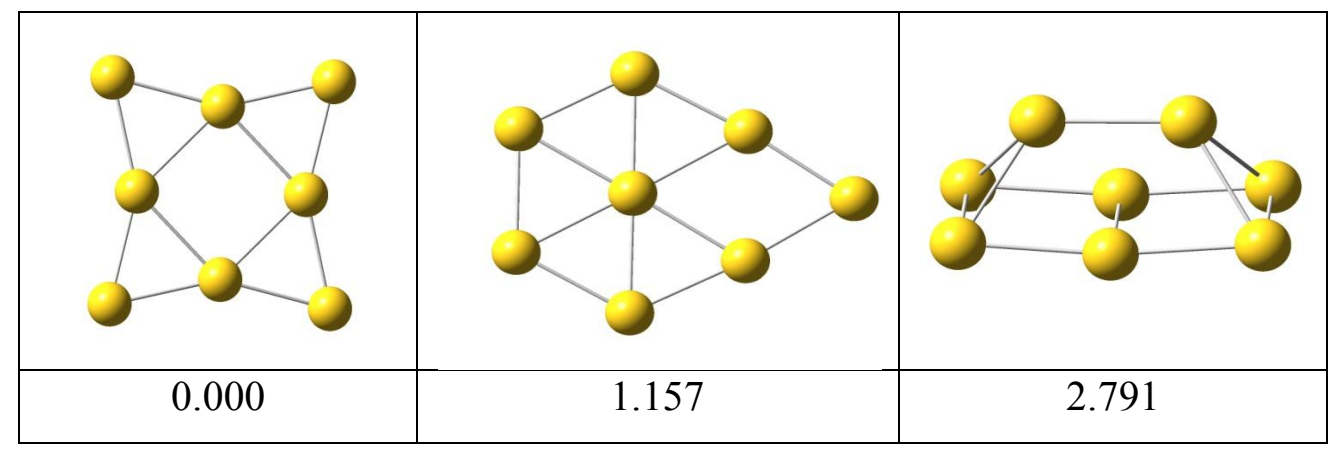

